\title{
Mechanical Behavior of Microelectromechanical Microshutters
}

\author{
Devin E. Burns, Justin S. Jones, Mary J. Li \\ NASA Goddard Space Flight Center, Greenbelt, MD
}

\begin{abstract}
A custom micro-mechanical test system was constructed using off-the-shelf components to characterize the mechanical properties of microshutters. Microshutters are rectangular microelectromechanical apertures which open and close about a narrow torsion bar hinge. Displacement measurements were verified using both capacitive and digital image correlation techniques. Repeatable experiments on $\mathrm{Si}_{3} \mathrm{~N}_{4}$ cantilever beams verified that the test system operates consistently. Using beam theory, the modulus of elasticity of the low stress $\mathrm{Si}_{3} \mathrm{~N}_{4}$ was approximately $150 \mathrm{GPa}$, though significant uncertainty exists for this measurement due primarily to imprecise knowledge of the cantilever thickness. Tests conducted on microshutter arrays concluded that reducing the $\mathrm{Si}_{3} \mathrm{~N}_{4}$ thickness from $250 \mathrm{~nm}$ to $500 \mathrm{~nm}$ reduces the torsional stiffness by a factor of approximately four. This is in good agreement with analytical and finite element models of the microshutters.
\end{abstract}

Keywords: Microshutters, MEMS, mechanical testing, silicon nitride, digital image correlation, James Webb Space Telescope

\section{INTRODUCTION}

Significant research and development conducted at NASA Goddard Space Flight Center has resulted in the production of flight ready microshutters arrays (MSA) ${ }^{1}$. The MSAs will serve as multi-object aperture selectors for the Near Infrared Spectrograph - one of three instruments planned for the James Webb Space Telescope (JWST). The specific requirements of JWST have prompted the use of an actuation scheme that uses both magnetic and electrostatic actuation to accomplish 2D addressing and latching of microshutters. While this has proven to be effective and reliable, extending the use of the MSA technology to future missions and into the commercial sector will require changing the actuation scheme. Specifically, the reliance on magnetic actuation, which requires a scanning permanent magnet, must be eliminated because it prevents scalability, adds mass and complexity, and is slow. Research has begun to design a Next Generation Microshutter Array (NGMSA) which can actuate, address, and latch shutters using only electrostatic forces. The development of NGMSAs promises to produce a modular and scalable solution with faster response times than MSAs.

Changes in the actuation scheme have necessitated changes in the geometry and materials used in NGMSA fabrication. As these changes are being implemented, a mechanical test system and mechanical models were developed to assist with design and process feedback. Of particular concern to successful NGMSA implementation, is obtaining a microshutter that is compliant enough to be actuated and latched with reasonable drive voltages. In the current study, a custom micromechanical test system was designed and tested for accuracy. This test system was then used to validate a finite element and an analytical model to establish a complimentary suite of tools for assessing microshutter performance and to eliminate uncertainty regarding new processing steps, materials, or geometrical changes.

\section{EXPERIMENTAL APPROACH}

\subsection{Microshutter Fabrication}

The microshutters consist of a torsion bar and a blade, as shown in Figure 1. The torsion bar is designed to allow the shutter to rotate 90 degrees through a well in the Si from a fully closed state (in which radiation is reflected) to a fully open state (in which radiation is transmitted). MSAs are fabricated from 4 inch silicon-on-insulator wafers using surface and bulk micromachining processes, as described in more detail elsewhere ${ }^{1,2}$. The microshutter consists of a low stress chemical vapor deposited $\mathrm{Si}_{3} \mathrm{~N}_{4}$ layer, which acts as the primary structural layer, a physical vapor deposited Al layer, which is primarily for electrical conductivity, and a thermally grown $\mathrm{SiO}_{2}$, which acts as an electrical insulator. In this study, shutters with nominal $\mathrm{Si}_{3} \mathrm{~N}_{4}$ thicknesses of $250 \mathrm{~nm}$ and $500 \mathrm{~nm}$ and $\mathrm{Al}$ thicknesses of $250 \mathrm{~nm}$ were fabricated. 
The nominal $250 \mathrm{~nm}$ and $500 \mathrm{~nm}$ low stress $\mathrm{Si}_{3} \mathrm{~N}_{4}$ depositions were performed by two separate external vendors who coated multiple wafers at one time as part of a batch process. The actual thicknesses of the wafers in each batch ranged between $246-249 \mathrm{~nm}$ (average $247 \mathrm{~nm}$ ) and $472-561 \mathrm{~nm}$ (average $515 \mathrm{~nm}$ ), respectively.

Test structures were fabricated in the same batch as the arrays for quality control and evaluation purposes. The test structures consisted of an array of cantilever beams $80 \mu \mathrm{m}$ wide with different lengths and material combinations.
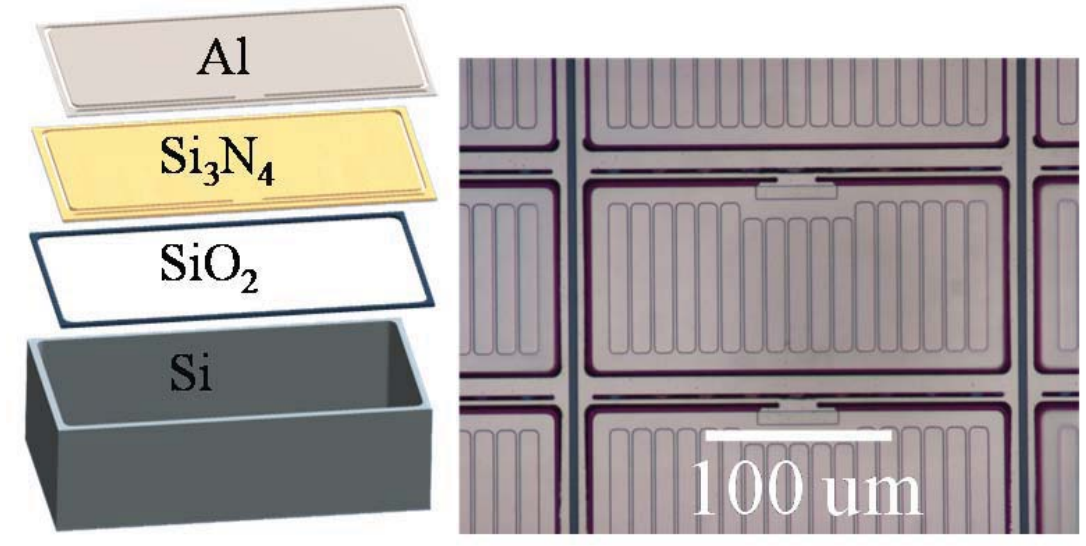

Figure 1. Fabrication layout of the microshutters (left) and normal view of the top MSA surface (right).

\subsection{Test System Components}

The micro-mechanical test system developed for this study was built using off-the-shelf components. A model and photograph of the test system are shown in Figure 2. The base structure for the test system consists of a breadboard ( 8 " $x 10$ "), onto which all the components were mounted. To the breadboard we mounted an x-y translation stage that was used for sample mounting and positioning. A vertically oriented translation stage (New Focus 9064) with a piezoelectric driven actuator capable of 30 nanometer step increments (Newport 8302) was positioned above the x-y translation stage to serve as the actuator. A metal block with a smooth surface finish was mounted to the top of the vertically-oriented translation stage to serve as a reference for the stage's position. A capacitive displacement gauge with nanometer resolution (MicroEpsilon capaNCDT 6500 with CS05 sensor) was then fixed in a position below the metal block, thus allowing for accurate measurement of the stage's position.

Two sets of optics were added to the test system to allow for precise alignment in both the $\mathrm{x}$ and $\mathrm{y}$ directions. A USB 3.0 CMOS machine vision camera (IDS-MicroEye UI-3480CP) with 8x telecentric lens (Edmund Optics 63-744) was added along the line of site that corresponded to the test specimens length so that an image could be taken at the start of the test for accurate determination of the probe location using digital imaging measurements. Frequently, a video of the test was also recorded so that any testing anomalies could be analyzed. A stereo microscope with variable magnification was added along a perpendicular line of site corresponding to the test specimen's width. The variable magnification was useful for identification of the test specimen of interest as well as positioning of the probe along the width direction. Finally, a capacitive comb-drive sensor (FemtoTools FT-S100) with a tungsten probe tip was mounted to the bottom of the vertically-mounted translation stage and served as the force sensor for the measurements. The force sensor has a $2 \mu \mathrm{m}$ wide wedge-shaped tip which probes the specimen and has a manufacturer's reported force-feedback sensitivity of $0.005 \mu \mathrm{N}$ and a full measurement range of $100 \mu \mathrm{N}$.

Fiber optic lighting was used to illuminate the test specimen. The entire test system was erected upon an optical table which was then placed on a base with passive dampers to help reduce vibration. An airflow-restricting enclosure was added to minimize the effect of air currents and thermal fluctuations in the room. The control and measurement hardware was all incorporated into a central LabVIEW program to facilitate testing and simplify data acquisition and processing. 


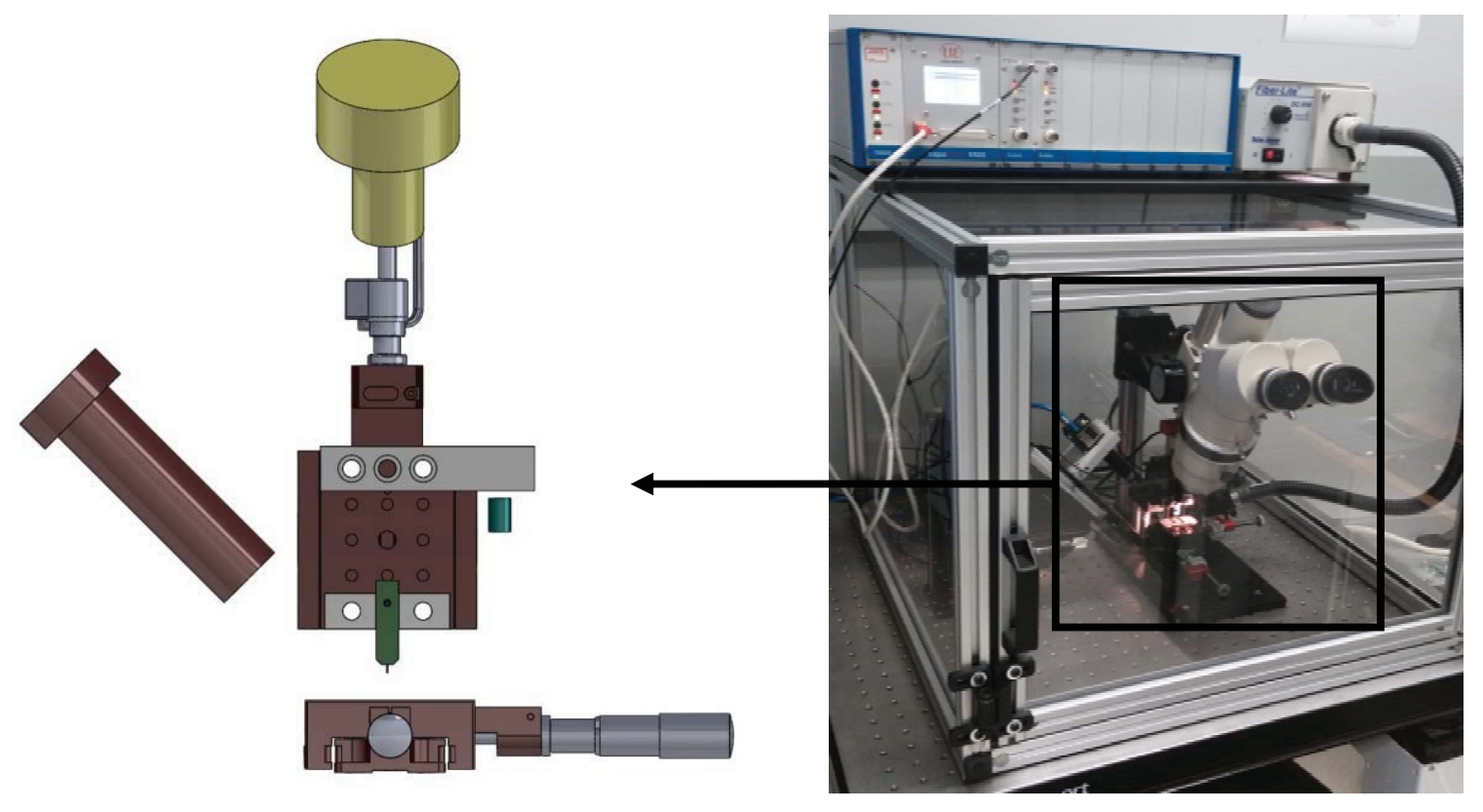

Figure 2: A model and photograph of the test system showing key system components for mechanical actuation and force measurement of microshutters. CMOS camera (left) views down from a 45-degree angle along the length direction; optical microscope (top) looks down from above to align along the width direction; x-y stage (lower) holds and positions the sample; actuator/stage and probe (center) engages the shutters; and capacitive gauge (center, small block below aluminum beam which is affixed to upper part of stage) records displacement.

\subsection{Test Procedures}

Tests of microshutters were performed at room temperature and with test operators several meters away from the test system to minimize vibration. The microshutter or test structure was first placed onto the lower $x-y$ stage of the test frame, beneath the force sensor/probe. Alignment of the array was performed using the CMOS camera and the optical system, such that the optical microscope view and the camera view (both from a downward facing viewpoint) are orthogonal to one another. This allows proper positioning of the probe tip at the desired location on the surface of the shutter (microscope shows position along width; camera shows position along length).

Lighting is adjusted using the orientation and intensity of the fiber optic source so that contrast is optimized for both the microscope and camera. Polarizing lenses on the lights help to reduce glare. Turning on the lights impart a small but measureable temperature change to the test region, so the system is allowed time to equilibrate before the force sensor is tared. Once this is done, the probe is then manually lowered (using the knob at the end of the actuator screw) until it just comes in contact with the test specimen. This event can be observed through the camera or microscope and is also detectible in the load data. From this location, the test is started through the control software and, quasi-static actuation is performed at a rate of $60 \mathrm{~nm} / \mathrm{second}$. Faster test speeds tended to increase system noise, perhaps due to natural frequencies of the system. The probe pressed downward into the specimen until either a desired load or displacement was achieved; each triggered using a software defined limit. The specimen was then unloaded at the same rate and then cycled either one or two more times to assess repeatability.

\section{RESULTS}

\subsection{Displacement Validation}

Both the force sensor and the capacitive displacement gauge are factory calibrated and guaranteed to be accurate within normal loading conditions. Given the load range, there was no means to verify the force sensor calibration in our laboratory so load measurements are assumed to be accurate to within the manufacturer's specifications. The capacitive sensor, as described above, measures the probe tip's translation indirectly by measuring the motion of the translation stage onto which the probe is mounted. Given that all components in this motion assembly (actuator, stage, and sensor) 
are very stiff compared to the test structures, there should be very little deviation in the displacement measurement due to compliance loss. Nevertheless, we thought it was important to assess the capacitance sensor's accuracy in case of losses in the force sensor's comb drive mechanism or flexing/shifting of the probe on the sample surface. This was achieved by performing a test on a butterfly test specimen and comparing measurements from the capacitance sensor with a secondary measurement. The butterfly test specimen is a test structure which consists of two opposing microshutters which pivot about a single torsion bar (analogous to a see-saw). The secondary displacement measurement was a commercial 3D digital image correlation (DIC) system with stated resolution down to tens of micrometers (microAramis from Trilion ${ }^{(\mathrm{R})}$ ). By orienting the entire testing system at a 60 -degree angle to the two cameras used for digital image correlation (as shown in Figure 3), the displacement of the test structure was measured simultaneously using digital imaging techniques and the capacitive method outlined above.

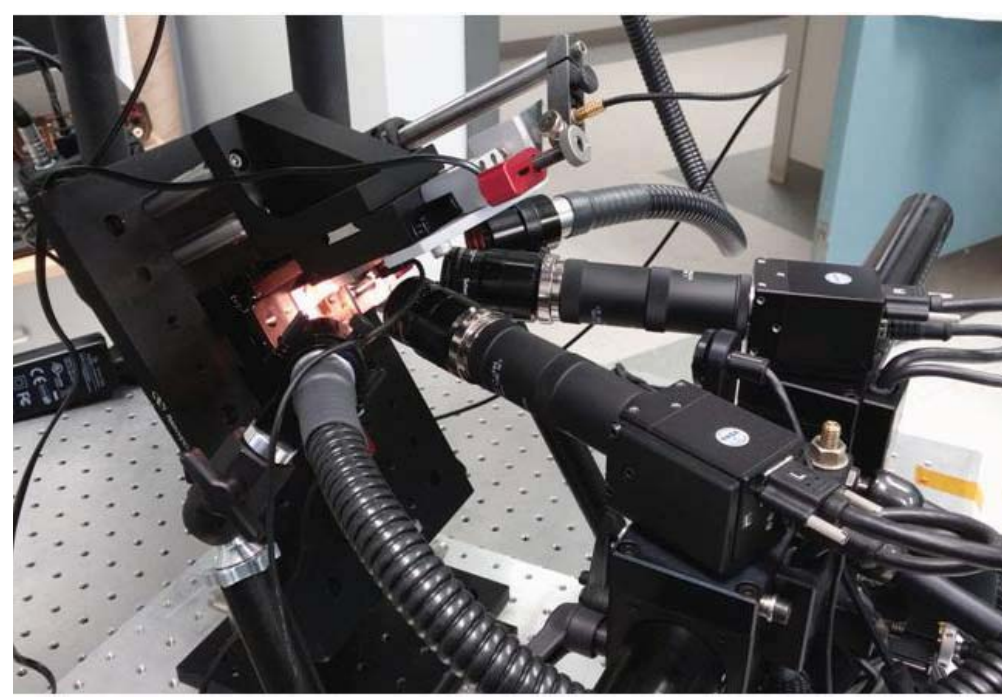

Figure 3. Setup for the verification of the capacitive displacement gauge using dual camera (stereo) DIC system (microAramis). Using this system, the position of a surface point very close to the probe tip could be tracked in 3D space.

The butterfly test structure was selected because the actuation and capacitive displacement measurement could be performed on one half of the test structure, while displacement verification via DIC could be performed on the opposite side of the test structure via DIC. In doing so, we assume that an applied displacement on one side would be equal and opposite at the same position on the opposite side of the butterfly. The left image in Figure 4 shows a close-up of a butterfly test structure, demonstrating the position of the probe tip as half of the structure is pressed downward. In order to use DIC, the otherwise uniform and specular surface of the test structures had to be coated to improve image tracking. An air brush filled with a mixture of ethanol and $2 \mu \mathrm{m} \mathrm{Al} \mathrm{O}_{3}$ particles was used to coat the test structures. The right image in Figure 4 shows the test structure array (with butterflies and several other kinds of test structures) with this speckle coating applied. The force probe can be seen faintly in the center, pressing on one of the butterfly structures. 


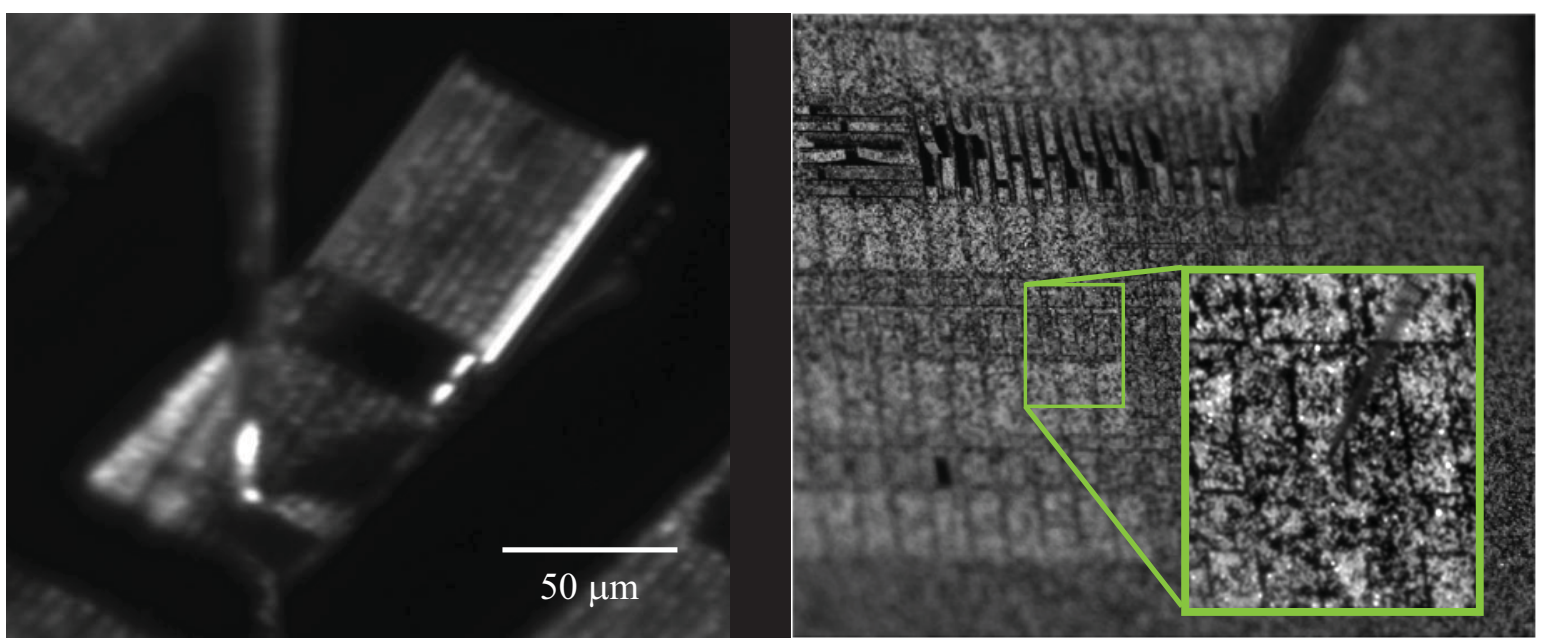

Figure 4. An example of a butterfly test structure during loading via the probe tip (left image) and a test structure array which was speckle-coated with alumina powder to improve DIC tracking, showing the probe engaging a butterfly (right image with inset).

As shown in Figure 5, excellent agreement was found between the two displacement measurements. This was repeated on several structures with similar results. While not presented here, the DIC system could offer valuable insight into other aspects of the test such as possible stress concentrations at the loading tip and asymmetric deformation; however, since the capacitive displacement sensor requires much less setup and sample preparation, it was the method used for the rest of the data presented in this study.

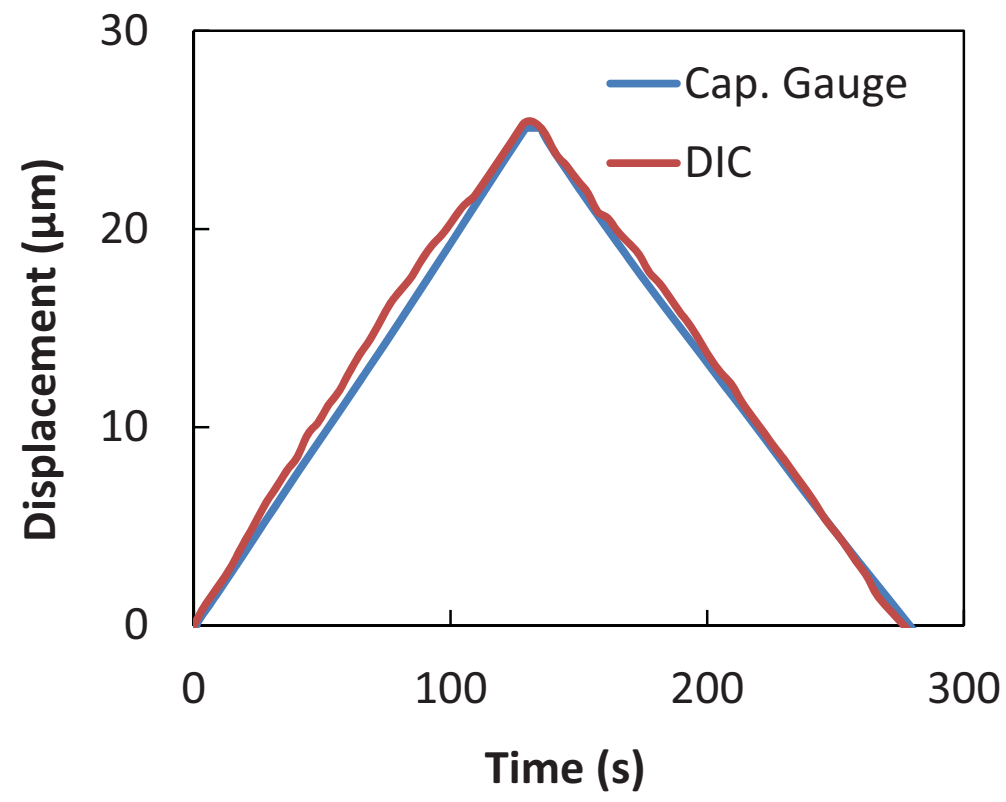

Figure 5. Displacement applied to a butterfly test structure measured by the capacitive displacement sensor and the digital image correlation system. 


\subsection{Cantilever Beam Experiments}

The cantilever beam test structures, fabricated alongside the microshutters, offered a good starting point for analysis of the reliability of the test system as well as an assessment of the mechanical properties of the low stress $\mathrm{Si}_{3} \mathrm{~N}_{4}$, which was necessary for modeling of the microshutters. Both the thick and thin cantilever beams were tested approximately $50 \mu \mathrm{m}$ from the fixed end of the cantilever to allow direct comparison between tests. Representative force vs. displacement curves for two thick and two thin cantilevers are shown in Figure 6. The average stiffness for the two thin cantilevers is $0.43 \mu \mathrm{N} / \mu \mathrm{m}$ and for the two thick cantilevers is $2.54 \mu \mathrm{N} / \mu \mathrm{m}$. The consistency between the two tests at each thickness suggests good reliability of the test system and procedure.

Beam theory can be used to approximate the Young's modulus of the cantilevers, but particular attention needs to be given to sources of error in the beam theory calculation. The largest contributor to the error is due to the uncertainty in the thickness of the $\mathrm{Si}_{3} \mathrm{~N}_{4}$ layer on each wafer and thus the thickness of the cantilever beams. Unfortunately, the exact $\mathrm{Si}_{3} \mathrm{~N}_{4}$ thickness for each individual wafer is unknown and thus the true cantilever thickness could range anywhere between the minimum and maximum values specified by the vendor. Using the minimum and maximum thickness as bounds, the measured Young's moduli range between 163 and $169 \mathrm{GPa}$ for the thin cantilever and 85 and $146 \mathrm{GPa}$ for the thick cantilevers. Clearly, the large range in modulus values, particularly for the thick cantilever data, creates uncertainty with respect to modeling and design of the microshutters and, in future work we intend to identify the $\mathrm{Si}_{3} \mathrm{~N}_{4}$ thickness at least on the wafer level for greater fidelity of our models. Reported modulus values of low stress $\mathrm{Si}_{3} \mathrm{~N}_{4}$ range between $95 \mathrm{GPa}$ and $370 \mathrm{GPa}$ with the majority of these measurements above $200 \mathrm{GPa}^{3-4}$. Bearing this in mind, we suspect the true modulus is likely at the upper end of our measured range and for consistency we chose to model both thin and thick microshutters with a modulus of elasticity of $150 \mathrm{GPa}$ for the $\mathrm{Si}_{3} \mathrm{~N}_{4}$.

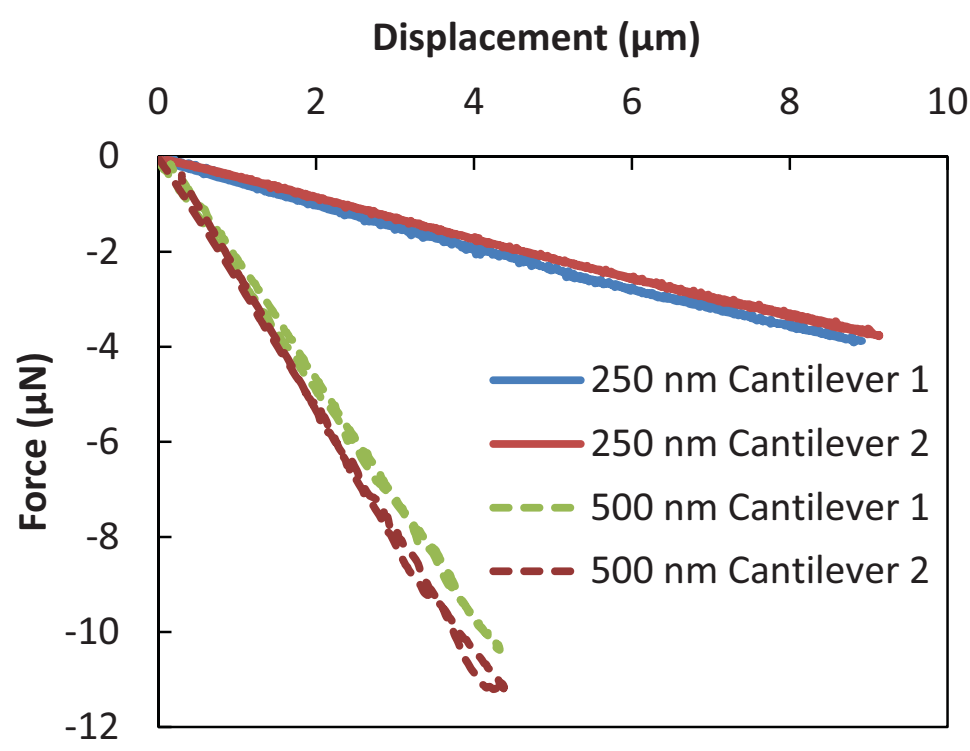

Figure 6. Quasi-static mechanical response of two $250 \mathrm{~nm}$ and two $500 \mathrm{~nm}$ thick $\mathrm{Si}_{3} \mathrm{~N}_{4}$ cantilever beams. All beams are $80 \mu \mathrm{m}$ wide and were tested approximately $50 \mu \mathrm{m}$ from the fixed end of the beams.

\subsection{Microshutter Experiments}

Achieving drive voltages compliant with flight specifications means designing microshutters with lower torsional stiffness than traditional magnetic actuated shutters. Both magnetic- and electrostatic-based microshutters were tested at the approximate geometric center of the shutter and the results of those tests are shown in Figure 7. As shown in the figure, the reduction in the $\mathrm{Si}_{3} \mathrm{~N}_{4}$ thickness from $500 \mathrm{~nm}$ to $250 \mathrm{~nm}$ results in a reduction of the torsional stiffness, from $0.061 \mu \mathrm{N} / \mu \mathrm{m}$ to $0.015 \mu \mathrm{N} / \mu \mathrm{m}$. This approximate factor of four reduction is consistent with finite element analysis (FEA) and analytical model predictions as shown by the agreement between the models and the data (Figure 7). The analytical 
model was based on a solution offered by Muskhelishvili ${ }^{5}$ for the torsional rigidity of a bilayer rectangular beam (the torsion bar, consisting of $\mathrm{Si}_{3} \mathrm{~N}_{4}$ and $\mathrm{Al}$ ). The finite element solution was a stationary solution developed in COMSOL and considered geometric nonlinearity effects. The better agreement between the FEA solution and the experimental data at higher displacements is likely due consideration of nonlinear effects. Both models assume an elastic modulus of 150 $\mathrm{GPa}$ for the $\mathrm{Si}_{3} \mathrm{~N}_{4}$ and $70 \mathrm{GPa}$ for the Al. The models also assume a nominal $\mathrm{Si}_{3} \mathrm{~N}_{4}$ thickness of $250 \mathrm{~nm}$ for the electrostatic array and $500 \mathrm{~nm}$ for the magnetic array supporting $200 \mathrm{~nm}$ of Al in both cases; deviations from these nominal values could significantly affect the model stiffness and effectiveness.

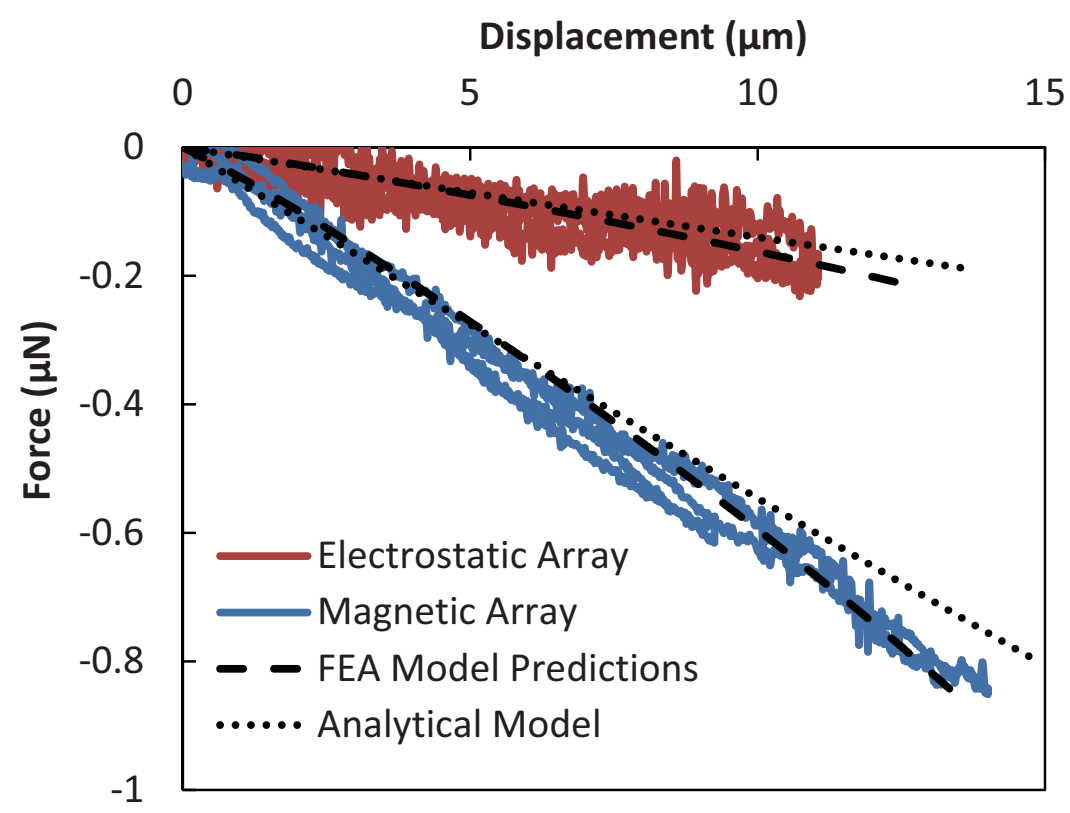

Figure 7. Torsional stiffnesses of electrostatically-actuated (250 nm $\left.\mathrm{Si}_{3} \mathrm{~N}_{4}, 200 \mathrm{~nm} \mathrm{Al}\right)$ and magnetically-actuated (500 nm $\mathrm{Si}_{3} \mathrm{~N}_{4}, 200 \mathrm{~nm} \mathrm{Al}$ ) microshutter arrays measured from quasi-static experiments. Also shown are analytical and finite element models predicting the torsional behavior.

\section{CONCLUSIONS}

In this work, we have described and demonstrated the construction of a custom micromechanical test system using offthe-shelf components that allows for accurate and efficient testing of extremely compliant microshutters and other test structures. Digital image correlation was used to independently verify the system's capacitive displacement gauge. Preliminary tests conducted on cantilever beams produced consistent results, supporting the reliability of the system components and testing procedure. Good agreement between the measured torsional stiffness of the microshutters and stiffnesses predicted by finite element and analytical models supports the accuracy of the test system and models. The verified models will now be used to guide future microshutter design changes.

\section{REFERENCES}

[1] Li, M.J., Brown, A.D., Kutyrev, A.S., Moseley, S.H., Mikula, V, "JWST Microshutter Array System and Beyond," Proc. SPIE 7594, 1-9 (2010).

[2] Li, M.J., Adachi, T., Allen, C.A., Babu, S.R., Bajikar, S., et al., "Microshutter Array System for James Webb Space Telescope," Proc. SPIE 6687, 1-13 (2007).

[3] Edwards, R.L., Coles, G., Sharpe, W.N., "Comparison of tensile and bulge tests for thin film silicon nitride," Exper. Mechan. 44, 49-54 (2004). 
[4] Kaushik, A., Kahn, H., Heuer, A.H., "Wafer-level mechanical characterization of silicon nitride MEMS," J. Microelectromech. Syst. 14(2), 359-367 (2005).

[5] Muskhelishvili, N.I., [Some Basic Problems of the Mathematical Theory of Elasticity] Noordhoff Ltd, Groningen, Netherlands, 623-626 (1963). 